\title{
Cost Savings of Human Milk as a Strategy to Reduce the Incidence of Necrotizing Enterocolitis in Very Low Birth Weight Infants
}

\author{
Tricia J. Johnson a, b Aloka L. Patel ${ }^{b, c}$ Harold R. Bigger ${ }^{c}$ Janet L. Engstrom ${ }^{b}$ \\ Paula P. Meier ${ }^{b, c}$
}

Departments of ${ }^{a}$ Health Systems Management, ${ }^{b}$ Women, Children and Family Nursing and ${ }^{c}$ Pediatrics, Rush University Medical Center, Chicago, III., USA

\section{Key Words}

Cost savings · Human milk · Necrotizing enterocolitis .

Very low birth weight infants

\begin{abstract}
Background: Necrotizing enterocolitis (NEC) is a costly morbidity in very low birth weight (VLBW; $<1,500 \mathrm{~g}$ birth weight) infants that increases hospital length of stay and requires expensive treatments. Objectives: To evaluate the cost of NEC as a function of dose and exposure period of human milk (HM) feedings received by VLBW infants during the neonatal intensive care unit (NICU) hospitalization and determine the drivers of differences in NICU hospitalization costs for infants with and without NEC. Methods: This study included 291 VLBW infants enrolled in an NIH-funded prospective observational cohort study between February 2008 and July 2012. We examined the incidence of NEC, NICU hospitalization cost, and cost of individual resources used during the NICU hospitalization. Results: Twenty-nine (10.0\%) infants developed NEC. The average total NICU hospitalization cost (in 2012 USD) was USD 180,163 for infants with NEC and USD 134,494 for infants without NEC $(p=0.024)$. NEC was associated with a marginal increase in costs of USD 43,818, after controlling for demographic characteristics, risk of NEC, and average daily dose of HM during days $1-14(p<0.001)$.
\end{abstract}

Each additional $\mathrm{ml} / \mathrm{kg} /$ day of HM during days 1-14 decreased non-NEC-related NICU costs by USD $534(p<0.001)$. Conclusions: Avoidance of formula and use of exclusive HM feedings during the first 14 days of life is an effective strategy to reduce the risk of NEC and resulting NICU costs in VLBW infants. Hospitals investing in initiatives to feed exclusive HM during the first 14 days of life could substantially reduce NEC-related NICU hospitalization costs.

두 2015 S. Karger AG, Base

\section{Introduction}

Very low birth weight (VLBW, $<1,500 \mathrm{~g}$ ) infants are susceptible to inflammation-based morbidities during neonatal intensive care unit (NICU) hospitalization that can lead to lifelong disability and high societal costs $[1,2]$. Necrotizing enterocolitis (NEC) is an inflammation-based morbidity [3] that affects 5-7\% of VLBW infants $[4,5]$, increasing the direct cost of the initial NICU hospitalization [4] and predisposing surviving infants to long-term complications $[1,6]$ and poor neurodevelopmental outcomes [7]. Preventive strategies that reduce the prevalence of NEC during the NICU hospitalization are both a clinical and an economic priority for society.

\section{KARGER 125}

(c) 2015 S. Karger AG, Base

$1661-7800 / 15 / 1074-0271 \$ 39.50 / 0$

E-Mail karger@karger.com

www.karger.com/neo
Tricia J. Johnson, $\mathrm{PhD}$, Associate Professor and Director Rush Center for the Advancement of Healthcare Value Department of Health Systems Management, Rush University Medical Center 1700 West Van Buren Street, TOB Suite 126B, Chicago, IL 60612 (USA)

E-Mail Tricia_J_Johnson@ rush.edu 


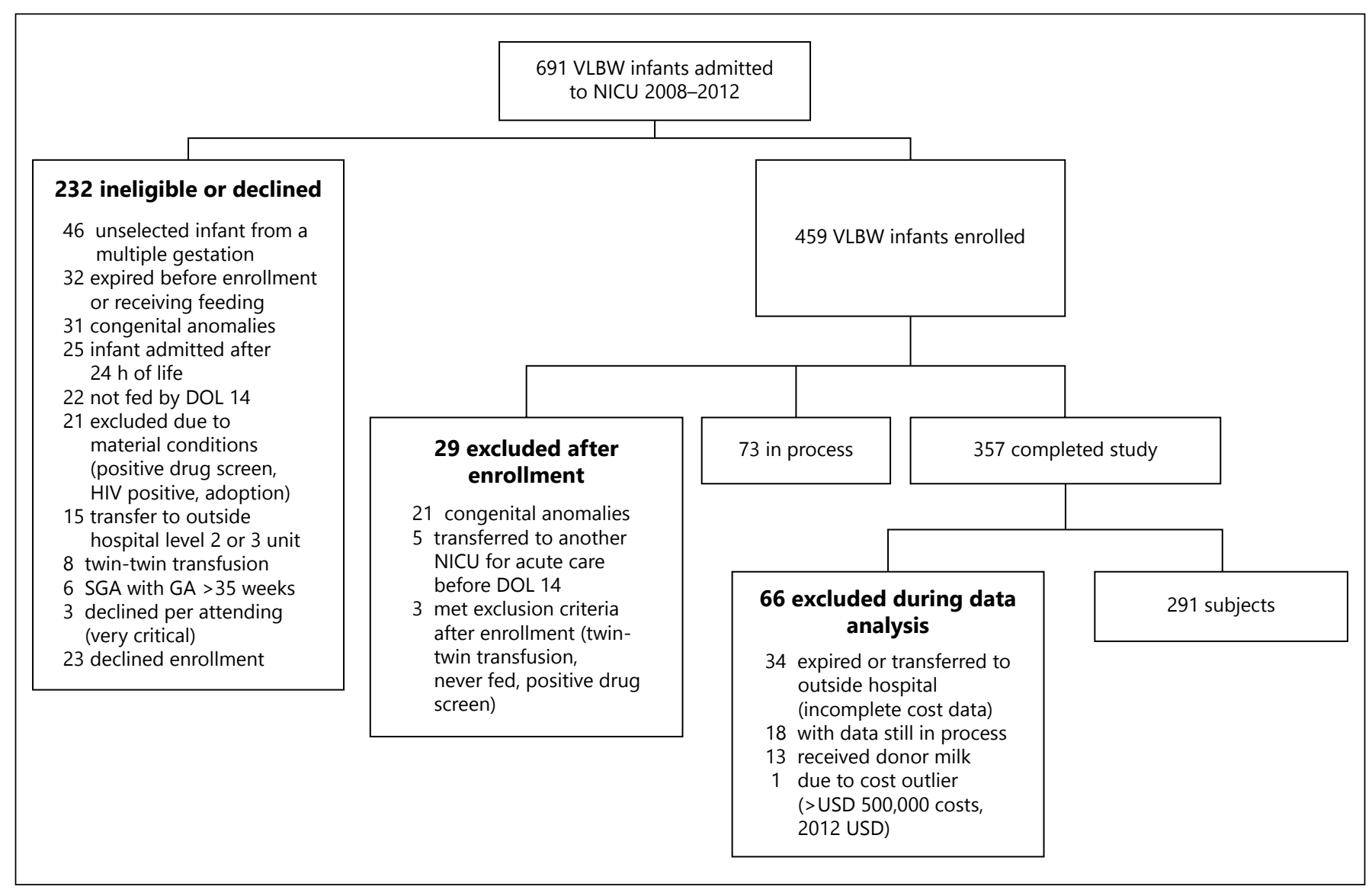

Fig. 1. Flow of infants into study. DOL = Day of life; $S G A=$ small for gestational age.

Several studies of VLBW infants have demonstrated a dose-response relationship between high doses of human milk (HM; milk from the infant's own mother, excludes donor $\mathrm{HM}$ ) received during NICU hospitalization and a reduction in the risk of NEC [8-10]. It remains unknown whether there is a specific amount of HM that must be received, whether bovine-based formulas should be avoided, or whether there is a critical postbirth period during which these interventions might be most important. No previous study has examined the dose and exposure period of HM feedings as a strategy to reduce the risk of NEC and its associated costs during the initial NICU hospitalization.

The purpose of this study was to examine the cost of NEC as a function of the dose and exposure period of HM feedings received by VLBW infants during NICU hospitalization and determine whether differences in costs for infants with and without NEC are due to longer lengths of hospital stay, more intensive use of hospital services, or both length of stay and service use.

\section{Methods}

This was an NIH-funded prospective cohort study of 291 VLBW infants cared for in the Rush University Medical Center (RUMC) NICU between February 2008 and June 2012 (fig. 1). The detailed inclusion/exclusion criteria for the study have been previously reported [11] and are summarized here. Inclusion criteria were birth weight $(\mathrm{BW})<1,500$ g, gestational age (GA) $<35$ weeks, enteral feedings initiated by day of life 14 , absence of major congenital anomalies or chromosomal disorders, and negative maternal drug screen. Infants were excluded from this analysis if they expired prior to NICU discharge or if they were transferred to a referral hospital prior to NICU discharge, as we did not have access to complete cost data for the NICU hospitalization. Although $98 \%$ of the enrolled infants received some HM from the mother, maternal initiation of lactation was not an inclusion criterion. The study was approved by the RUMC Institutional Review Board, and informed consent was obtained from the parent or legal guardian.

NEC was defined according to modified Bell's criteria $\geq 2$ [12], with infants meeting both clinical (abdominal distension, feeding intolerance, bloody stool, abdominal tenderness, or bilious residuals) and radiographic (pneumatosis intestinalis, portal venous gas, or pneumoperitoneum) criteria for NEC. Cases of spontaneous intestinal perforation, defined as isolated bowel perforation within 
the first-week postbirth period with no pneumatosis intestinalis or portal venous gas [13], were not included in the NEC cases. Surgically managed NEC cases were defined as infants who received a peritoneal drain or underwent exploratory laparotomy for treatment of NEC. All other cases of NEC were considered medically managed.

Direct and indirect costs for the NICU hospitalization were collected from RUMC's system-wide cost accounting system using previously published procedures $[4,11]$. Costs were adjusted to 2012 USD using the Bureau of Labor Statistics Consumer Price Index for all items [14].

Dose and exposure period of HM feedings were calculated using techniques described in our previous studies $[11,15]$. Dose of $\mathrm{HM}$ was calculated as both a weight-adjusted dose $(\mathrm{ml} / \mathrm{kg} /$ day $)$ and percentage of enteral feedings equal to HM. The average dose of $\mathrm{HM}$, in $\mathrm{ml} / \mathrm{kg} /$ day (HM-DD) and as a percentage of total enteral feedings (HM-PCT), was calculated for days 1-14 [15]. The day 1-14 exposure period was selected based on the critical transition from intrauterine to extrauterine nutrition and to avoid reverse causality in infants who developed NEC. Feedings were initiated with unfortified HM or preterm formula (20 calories/ounce). All infants received HM from their own mothers. HM feedings were fortified according to a standardized protocol [11]. Formula feedings were used only when adequate volumes of HM were unavailable for feedings. Three additional feeding variables were included: a binary variable indicating whether the infant had received any formula in days $1-14$, a binary variable indicating whether the infant had received any bovine human milk fortifier (HMF) in days 1-14, and a continuous variable for the number of days to full feeding (number of days from first feeding to the first day an infant received $\geq 120 \mathrm{ml} / \mathrm{kg} /$ day of enteral nutrition and no intravenous fluids).

Infant and maternal factors included as covariates were infant BW (g), GA (completed weeks), gender, days of initial antibiotic use (none, 1-4 days, $\geq 5$ days) [16], small for gestational age (defined as BW below the 10th percentile according to Olsen et al. [17]), and maternal race (Black/African-American, Hispanic, White, or other race). An additional covariate accounted for two quality improvement (QI) initiatives targeted at decreasing the incidence of NEC (enteral feeding protocol and nasogastric tube maintenance guidelines) during the study period. The QI phases were categorized as baseline (June 2009 - November 2009), QI phase 1 (December 2009 - May 2010), and QI phase 2 (June 2010 - June 2012) [18].

\section{Statistical Analysis}

Two independent-samples $t$ tests, one-way analysis of variance, and $\chi^{2}$ tests were conducted to compare the characteristics of infants with and without NEC. Direct costs were compared using two independent-samples t tests. A binary logistic regression model was fit, and the predicted values from the model were used to create a propensity score as a composite score for the risk of NEC $[11,19]$. The model included demographic variables (race, gender, BW, small for GA), QI intervention phase, days to full feeding, any formula in days $1-14$, and any HMF in days 1-14. An interaction term between BW and days to full feeding was included to account for a potential nonlinear relationship between these two variables in predicting the risk of NEC.

A generalized linear regression model was estimated with a log link function and gamma distribution for NICU hospitalization cost with BW, race, gender, HM-DD in days $1-14$, NEC, and NEC
Table 1. Description of sample $(n=291)$

\begin{tabular}{|c|c|c|c|}
\hline Variable & $\begin{array}{l}\text { With } \\
\text { NEC } \\
(n=29)\end{array}$ & $\begin{array}{l}\text { Without } \\
\text { NEC } \\
(n=262)\end{array}$ & $\mathrm{p}$ \\
\hline BW, g & $1,050 \pm 200$ & $1,065 \pm 261$ & 0.768 \\
\hline GA, weeks & $27.7 \pm 2.2$ & $28.2 \pm 2.4$ & 0.231 \\
\hline Male & $24(83)$ & $132(50)$ & $<0.001$ \\
\hline QI phase & & & $<0.001$ \\
\hline $\begin{array}{c}\text { Baseline (February 1, 2008 - } \\
\text { November 30, 2009) }\end{array}$ & $17(59)$ & $128(49)$ & \\
\hline $\begin{array}{l}\text { Phase } 1 \text { (December } 1,2009- \\
\text { May } 31,2010)\end{array}$ & $9(31)$ & $25(10)$ & \\
\hline $\begin{array}{c}\text { Phase } 2 \text { (June 1, } 2010- \\
\text { October 31, 2012) }\end{array}$ & $3(10)$ & $109(42)$ & \\
\hline Duration of initial antibiotic course & & & 0.351 \\
\hline 0 days & $0(0)$ & $15(6)$ & \\
\hline 1-4 days & $12(41)$ & $116(44)$ & \\
\hline $5+$ days & $17(59)$ & $131(50)$ & \\
\hline Maternal race & & & 0.936 \\
\hline Black/African-American & $16(55)$ & $137(52)$ & \\
\hline White/other & $5(17)$ & $52(20)$ & \\
\hline Hispanic & $8(28)$ & $73(28)$ & \\
\hline Small for GA & $4(14)$ & $62(24)$ & 0.228 \\
\hline Days to full feeding & $21.7 \pm 22.0$ & $15.2 \pm 10.4$ & 0.132 \\
\hline DOL $1-14$ average $\mathrm{ml} / \mathrm{kg} /$ day HM & $25.7 \pm 17.9$ & $30.0 \pm 28.1$ & 0.254 \\
\hline DOL $1-14$ cumulative percent HM & $80 \pm 31$ & $81 \pm 33$ & 0.943 \\
\hline Any formula by DOL 14 & $14(48)$ & $99(38)$ & 0.271 \\
\hline $\begin{array}{l}\text { DOL of first formula, for infants } \\
\text { with formula by DOL } 14\end{array}$ & $6.9+3.6$ & $6.9+4.2$ & 0.992 \\
\hline Any HMF by DOL 14 & $11(38)$ & $95(36)$ & 0.859 \\
\hline Formula and HMF by DOL 14 & $8(28)$ & $41(16)$ & 0.103 \\
\hline
\end{tabular}

Values are means $\pm \mathrm{SD}$ or $\mathrm{n}(\%) . \mathrm{DOL}=$ Day of life.

propensity score. The mean-variance relationship was selected using a modified Park test [20]. We computed the marginal economic effect of each variable based on the mean predicted cost of NICU hospitalization [21]. Statistical analyses were conducted in SAS Version 9.2 (Cary, N.C., USA). Statistical significance was set at $\mathrm{p}<$ 0.05 .

\section{Results}

Of the 291 infants in the analysis, 29 (10.0\%) developed NEC at a median postnatal age of 24 days. There were no differences in HM-DD, proportion of infants exposed to formula, or proportion of infants exposed to bovine $\mathrm{HMF}$ in days $1-14$ (table 1 ).

The mean total NICU hospitalization cost was USD $180,163 \pm 100,824$ for infants with NEC and USD $134,494 \pm 72,604$ for infants without NEC ( $\mathrm{p}=0.024$; table 2). Infants with NEC had longer lengths of stay $(\mathrm{p}=0.022)$ and higher NICU hospital costs per day $(\mathrm{p}<$ 
Table 2. Comparison of direct cost categories by presence of NEC, in 2012 USD

\begin{tabular}{lccr}
\hline Variable & With NEC $(\mathrm{n}=29)$ & Without NEC $(\mathrm{n}=262)$ & $\mathrm{p}$ \\
\hline NICU length of stay & $85 \pm 36$ & $70 \pm 33$ & 0.022 \\
NICU total costs & $180,163 \pm 100,824$ & $134,494 \pm 72,604$ & 0.024 \\
NICU total costs per day & $2,032 \pm 175$ & $1,874 \pm 170$ & $<0.001$ \\
NICU total direct costs & $111,398 \pm 68,188$ & $80,717 \pm 48,028$ & 0.025 \\
NICU room and board & $82,974 \pm 455,551$ & $65,414 \pm 34,991$ & 0.053 \\
Pharmacy & $11,900 \pm 11,830$ & $5,080 \pm 4,194$ & 0.005 \\
Respiratory therapy & $6,815 \pm 6,677$ & $4,395 \pm 6,073$ & 0.045 \\
Laboratory and pathology & $5,058 \pm 3,756$ & $2,698 \pm 2,053$ & 0.002 \\
Diagnostics & $2,483 \pm 1,807$ & $1,188 \pm 1,246$ & $<0.001$ \\
Surgery & $1,042 \pm 1,880$ & $673 \pm 1,351$ & 0.313 \\
Cardiology & $511 \pm 558$ & $496 \pm 667$ & 0.904 \\
Other therapies (speech, physical) & $393 \pm 353$ & $423 \pm 331$ & 0.647 \\
Other services & $232 \pm 558$ & $348 \pm 1,294$ & 0.176 \\
NICU total indirect costs & $68,765 \pm 39,307$ & $53,777 \pm 40,019$ & 0.056 \\
\hline
\end{tabular}

Values are means \pm SD.

Table 3. Relationship between HM and NEC: results from binary logistic regression model

\begin{tabular}{llll}
\hline Variable & Coefficient & Odds ratio (95\% CI) & $\mathrm{p}$ \\
\hline Any formula by DOL 14 & 1.245 & $3.47(1.22-9.92)$ & 0.020 \\
Any HMF by DOL 14 & 0.565 & $1.76(0.59-5.26)$ & 0.311 \\
BW & -0.071 & $0.93(0.74-1.18)$ & 0.550 \\
Small for GA & -0.431 & $0.65(0.17-2.44)$ & 0.523 \\
Days to full feed & 0.091 & $1.10(1.02-1.18)$ & 0.015 \\
BW $\times$ days to full feed interaction (centered) & 0.0005 & - & 0.001 \\
c-statistic & & 0.855 & \\
\hline
\end{tabular}

For infants with NEC and days to full feed that exceeded day of onset of NEC, days to full feed set equal day of onset of NEC $(n=5)$ less (DOL of full feed - days to full feed). Model controls for race/ethnicity, gender, and $\mathrm{QI}$ phase. $\mathrm{DOL}=$ Day of life.

$0.001)$. Of the 29 infants with NEC, $5(17 \%)$ were surgically managed. The mean NICU costs were more than twice as high for infants with surgically versus medically managed NEC (USD $313,745 \pm 147,237$ vs. USD $152,333 \pm 62,593)$. The direct costs of NICU hospitalization were USD 30,681 higher for infants with NEC (table 2). The largest portion of costs was for room and board, representing $74.5 \%$ of the direct costs for infants with NEC and $81.0 \%$ of the direct costs for infants without NEC (USD 82,974 vs. USD 65,414, $\mathrm{p}=0.053$ ). For infants with NEC, pharmacy costs were 2.3 times higher $(\mathrm{p}=0.005)$, respiratory therapy costs were 1.6 times higher $(\mathrm{p}=0.045)$, and laboratory and pathology costs were 1.9 times higher $(p=0.002)$ compared to infants without NEC.
In the regression analysis for the risk of NEC, infants receiving any formula feedings during days $1-14$ had 3.5 times greater risk of developing NEC $(\mathrm{p}=0.020)$ (table 3$)$. While days to achieve full feedings increased the risk of NEC, the interaction between BW and days to full feedings was also associated with an increased risk of NEC, indicating that larger infants with delayed achievement of full feedings were at an even greater risk of NEC than were smaller infants.

After controlling for other variables, NEC was associated with USD 43,818 additional costs for the NICU hospitalization ( $\mathrm{p}<0.001$; table 4$)$. After controlling for NEC, each additional $\mathrm{ml} / \mathrm{kg} /$ day of HM in days $1-14$ was associated with a reduction of USD 534 in non-NEC-related NICU costs $(p<0.001)$. The propensity score for NEC 
Table 4. Relationship between HM, NEC, and total costs: results from the generalized linear regression model

\begin{tabular}{llr}
\hline & Marginal effect & $\mathrm{p}$ \\
\hline Propensity score for NEC & -34 & 0.906 \\
NEC & 43,818 & $<0.001$ \\
DOL 1-14 average ml/kg/day HM & -534 & $<0.001$ \\
$\quad$ (per 1-ml/kg/day increment) & & \\
\hline
\end{tabular}

Model controls for BW, race/ethnicity, and gender.

was not associated with costs, suggesting the model had no undetected heterogeneity related to the risk factors for NEC that might be associated with costs.

\section{Discussion}

To our knowledge, this is the first prospective study to evaluate the impact of formula and HM intake during days 1-14 on the risk of NEC, NEC-related hospital costs, and non-NEC-related hospital costs in a cohort of VLBW infants. Exposure to any amount of formula during days 1-14 increased the risk of NEC more than threefold. NEC substantially increased the cost of care, accounting for an additional USD 43,818 per infant in NICU hospitalization costs. These costs were due not only to a longer NICU stay, but also to greater per diem resource use. This perinfant hospital cost translated into an additional USD 1.27 million for the cohort of 291 infants in our study. These cost data likely underestimate the societal burden of NEC because we did not measure the potential loss of productive years and our analyses excluded infants who expired $(n=3)$, including those due to NEC.

Our study adds to other human and animal research that suggests the transition from intrauterine to extrauterine nutrition, captured in the exposure period of days $1-14$, is an especially vulnerable time for VLBW infants, with respect to the risk of NEC and perhaps other inflammation-based morbidities [10, 15, 22]. To our knowledge, ours is the first clinical study to demonstrate that early exposure to bovine-based infant formula - rather than a specific HM-DD or a threshold HM-PCT - increases the risk of NEC. Other human and animal studies have demonstrated that bovine-based formula significantly increases intestinal permeability, with the potential for translocation of pathogens and toxins through the open paracellular pathways in the epithelial lining of the gut to the underlying mucosa [23], upregulates inflammatory and oxidative stress processes in the gut [24], and is directly toxic to gut epithelial cells [25]. The fact that even small amounts of formula can increase the risk of NEC in a mostly HM-fed cohort is supported by numerous biologic mechanisms [25].

Although our study did not find a statistical relationship between the risk of NEC and combined impact of HMF and formula for days 1-14, this relationship trended toward significance. Several factors unique to our clinical feeding protocol may have masked this combined effect and warrant further study. First, only 38 and $36 \%$ of infants with and without NEC, respectively, had received HMF by day 14 due to our feeding advancement protocol that extends fortifier-free colostrum feedings until infants approach full enteral feedings. Second, only 28 and $16 \%$ of infants with and without NEC received both HMF and formula during days 1-14, so these numbers may have been too small to detect a significant relationship. Third, the QI intervention implemented in December 2009 specifically delayed fortification until after the infant reached full volume feedings [18]. Thus, in controlling for the QI phase, we may have statistically controlled for the impact of fortifier.

While it is apparent that NEC is a costly morbidity, it is difficult to compare the magnitude of our findings with previous studies due to the differences in methodologies used to calculate hospital costs. Our study used microlevel cost data while Ganapathy et al. [26] estimated direct costs based on cost-to-charge ratios. Their study included infants with $\mathrm{BW} \leq 1,250 \mathrm{~g}$, and their analyses did not control for infant BW or GA, both of which are significant contributors to hospital costs. In our previous research, NEC was associated with an incremental cost of USD 15,440 (in 2010 USD) per infant, after controlling for sociodemographic characteristics and the presence of other morbidities [4]. However, the findings from this study did not control for the presence of other morbidities, so the cost of NEC may be higher due to the fact that infants with NEC are at an increased risk for other morbidities [4]. Bisquera et al. [27] compared hospital charges and length of hospital stay for infants with and without NEC born between 1992 and 1994. Since only hospital charges and not costs were reported, it is difficult to compare their economic data to ours or to those who reported cost-tocharge ratios.

After controlling for NEC, we found a significant independent effect between the dose of HM received during days 1-14 and NICU hospitalization cost, with each additional $\mathrm{ml} / \mathrm{kg} /$ day of $\mathrm{HM}$ reducing hospital costs by USD 534. A single standard deviation increase in the 
HM-DD during days 1-14 was associated with USD 9,559 lower NICU hospitalization costs per infant, independent of the presence of NEC. Since our propensity score controlled for multiple risk factors for NEC, including BW, gender, and days to reach full enteral feedings, it is unlikely that these additional non-NEC cost savings are due to unaccounted confounding variables. We speculate that the independent impact of HM-DD in days $1-14$ is due to a lower risk of other common prematurity-related morbidities, including late-onset sepsis [11] and chronic lung disease [28], that were mitigated by high doses of HM during this early critical exposure period. Days 1-14 may be a critical period for VLBW infants when high doses of HM should be prioritized, both as a mechanism to reduce the risk of NEC and overall NICU hospital costs.

\section{Funding}

This study was funded by NIH grant NR010009 (PI: Meier) and the Rush University Scientific Leadership Committee.

\section{References}

1 Johnson TJ, Patel AL, Bigger HR, Engstrom JL, Meier PP: Economic benefits and costs of human milk feedings: a strategy to reduce the risk of prematurity-related morbidities in very low birth weight infants. Adv Nutr 2014; 5:207-212.

2 Schultz C, Temming P, Bucsky P, Gopel W, Strunk T, Hartel C: Immature anti-inflammatory response in neonates. Clin Exp Immunol 2004;135:130-136.

3 McElroy SJ, Underwood MA, Sherman MP: Paneth cells and necrotizing enterocolitis: a novel hypothesis for disease pathogenesis. Neonatology 2013;103:10-20.

4 Johnson TJ, Patel AL, Jegier B, Engstrom JL, Meier PP: The cost of morbidities in very low birth weight infants. J Pediatr 2013;162:243249.

5 Horbar JD, Carpenter JH, Badger GJ, Kenny MJ, Soll RF, Morrow KA, Buzas JS: Mortality and neonatal morbidity among infants 501 to 1500 grams from 2000 to 2009. Pediatrics 2012;129:1019-1026.

6 Ganapathy V, Hay JW, Kim JH, Lee ML, Rechtman DJ: Long term healthcare costs of infants who survived neonatal necrotizing enterocolitis: a retrospective longitudinal study among infants enrolled in Texas Medicaid. BMC Pediatr 2013;13:127.

7 Schulzke SM, Deshpande GC, Patole SK: Neurodevelopmental outcomes of very lowbirth-weight infants with necrotizing enterocolitis: a systematic review of observational studies. Arch Pediatr Adolesc Med 2007;161: 583-590.

8 Meinzen-Derr J, Poindexter B, Wrage L, Morrow AL, Stoll B, Donovan EF: Role of human milk in extremely low birth weight infants' risk of necrotizing enterocolitis or death. J Perinatol 2009;29:57-62.

9 Sisk PM, Lovelady CA, Dillard RG, Gruber $\mathrm{KJ}$, O'Shea TM: Early human milk feeding is associated with a lower risk of necrotizing enterocolitis in very low birth weight infants. J Perinatol 2007;27:428-433.
10 Corpeleijn WE, Kouwenhoven SM, Paap MC, van Vliet I, Scheerder I, Muizer Y, et al: Intake of own mother's milk during the first days of life is associated with decreased morbidity and mortality in very low birth weight infants during the first 60 days of life. Neonatology 2012;102:276-281.

11 Patel AL, Johnson TJ, Engstrom JL, Fogg LF, Jegier BJ, Bigger HR, Meier PP: Impact of early human milk on sepsis and health care costs in very low birthweight infants. J Perinatol 2013;33:514-519.

12 Walsh MC, Kliegman RM: Necrotizing enterocolitis: treatment based on staging criteria. Pediatr Clin North Am 1986;33:179-201.

13 Gordon PV, Clark R, Swanson JR, Spitzer A: Can a national dataset generate a nomogram for necrotizing enterocolitis onset? J Perinatol 2014;34:732-735.

14 Consumer price index for all items, 20082010. Bureau of Labor Statistics, United States Department of Labor. http://www.bls.gov/ data/ (accessed February 19, 2012).

15 Bigger HR, Fogg LJ, Patel A, Johnson T, Engstrom JL, Meier PP: Quality indicators for human milk use in very low-birthweight infants: are we measuring what we should be measuring? J Perinatol 2014;34:287-291.

16 Cotten CM, Taylor S, Stoll B, Goldberg RN, Hansen NI, Sanchez PJ, et al: Prolonged duration of initial empirical antibiotic treatment is associated with increased rates of necrotizing enterocolitis and death for extremely low birth weight infants. Pediatrics 2009;123:5866.

17 Olsen IE, Groveman SA, Lawson ML, Clark $\mathrm{RH}$, Zemel BS: New intrauterine growth curves based on United States data. Pediatrics 2010;125:e214-e224.
18 Patel AL, Trivedi S, Parikh NM, Ruf A, Scala $\mathrm{CM}$, Witowich $\mathrm{G}$, et al: Reducing necrotizing enterocolitis in very low birth weight infants using quality improvement methods. J Perinatol 2014;34:850-857.

19 Rosenbaum PR, Rubin DB: The central role of the propensity score in observational studies for causal effects. Biometrika 1983;70:41-55.

20 Manning WG, Mullahy J: Estimating log models: to transform or not to transform? J Health Econ 2001;20:461-494.

21 Jones AM: Applied Econometrics for Health Economists: A Practical Guide, ed 2. London, Radcliffe Publishing, 2007.

22 Sullivan S, Schanler RJ, Kim JH, Patel AL, Trawöger R, Kiechl-Kohlendorfer U, et al: An exclusively human milk-based diet is associated with a lower rate of necrotizing enterocolitis than a diet of human milk and bovine milkbased products. J Pediatr 2010;156:562-567.e1.

23 Taylor SN, Basile LA, Ebeling M, Wagner CL: Intestinal permeability in preterm infants by feeding type: mother's milk versus formula. Breastfeed Med 2009;4:11-15.

24 Sangild PT: Gut responses to enteral nutrition in preterm infants and animals. Exp Biol Med 2006;231:1695-1711.

25 Penn AH: Digested formula but not digested fresh human milk causes death of intestinal cells in vitro: implications for necrotizing enterocolitis. Pediatr Res 2012;72:560-567.

26 Ganapathy V, Hay JW, Kim JH: Costs of necrotizing enterocolitis and cost-effectiveness of exclusively human milk-based products in feeding extremely premature infants. Breastfeed Med 2012;7:29-37.

27 Bisquera JA, Cooper TR, Berseth CL: Impact of necrotizing enterocolitis on length of stay and hospital charges in very low birth weight infants. Pediatrics 2002;109:423-428.

28 Schanler RJ, Lau C, Hurst NM, Smith EOB: Randomized trial of donor human milk versus preterm formula as substitutes for mothers' own milk in the feeding of extremely premature infants. Pediatrics 2005;116:400-406. 